SDM 2011 Student Papers Competition

\title{
Design of Fiber-steered Variable-stiffness Laminates Based on a Given Lamination Parameters Distribution
}

\author{
Julien M.J.F. van Campen*, Christos Kassapoglou’, Zafer Gürdal ${ }^{\ddagger}$ \\ Aerospace Structures, Delft University of Technology, \\ Kluyverweg 1, 2629 HS Delft, the Netherlands
}

\begin{abstract}
Mechanical properties of fiber-reinforced laminated composite materials are directionally dependent. Contemporary laminated composite design aims to make effective use of these directional properties by means of stacking sequence design, selecting the fiber orientation angle of each ply from a predefined set. Automated fiber-placement (AFP) technology can be used to improve the efficacy of composite materials by means of fiber steering. The variation of fiber orientation angles per ply of the laminate yields a variable stiffness (VS) laminate. For optimization purposes it is attractive to design such laminates in terms of lamination parameters (LP), as the number of design variables per point in the structure can be reduced to as little as four dimensionless variables considering balanced symmetric layups, and because many lay-up optimization problems can be made convex by describing them in terms of LPs. VS laminate design in terms of LP requires the obtained LP distribution to be converted into an actual fiber angle design. In a previous study the authors proposed a method to convert VS laminate designs using LPs into fiber angle designs. This method includes a constraint on in-plane curvature, a manufacturing constraint related to AFP. Thickness build-up will occur due to fiber steering. The amount of thickness build-up that results from the obtained fiber angle designs is discussed here as a function of the constraint on fiber curvature. The streamline analogy is used to obtain an estimate for thickness build-up and to determine fiber paths. A square plate loaded in biaxial compression is used to demonstrate the effect of the in-plane curvature constraint on thickness build-up, and several fiber angle designs, thickness distributions and fiber paths are given for this structure.
\end{abstract}

\section{Nomenclature}

$\begin{array}{lll}\alpha & = & \text { step size } \\ \beta & = & \text { penalty term } \\ \kappa & = & \text { in-plane curvature } \\ \mathbf{V} & = & \text { lamination parameters } \\ \Phi & = & \text { augmented objective } \\ \Psi & = & \text { stream function } \\ \varepsilon & = & \text { bonus term } \\ C & = & \text { constant } \\ t & = & \text { thickness } \\ V_{i}^{\mathbf{A}} & = & \text { lamination parameters for A-matrix } \\ V_{i}^{\mathbf{D}} & = & \text { lamination parameters for D-matrix } \\ \Gamma & = & \text { matrices of material invariants } \\ \mathbf{g} & = & \text { gradient } \\ \mathbf{n} & = & \text { normal vector }\end{array}$

*PhD Student, Aerospace Structures, Delft University of Technology, Member AIAA, j.m.j.f.vancampen@tudelft.nl.

${ }^{\dagger}$ Associate Professor, Aerospace Structures, Delft University of Technology, Member AIAA.

${ }_{\ddagger}^{\ddagger}$ Professor, Aerospace Structures, Chair Holder, Delft University of Technology, Associate Fellow AIAA. 


$\begin{array}{lll}\mathbf{s} & = & \text { tangent vector } \\ \theta & = & \text { fiber angle } \\ f & = & \text { objective } \\ g & = & \text { constraint margin } \\ n & = & \text { number of layers in the laminate } \\ n_{d} & = & \text { number of layers in the laminate for which the orientation is designed } \\ S & = & \text { reduced stiffness } \\ U_{i} & = & \text { material invariant } \\ \text { AFP } & = & \text { automated fiber placement } \\ \text { CLT } & = & \text { classical lamination theory } \\ \text { CS } & = & \text { constant stiffness } \\ \text { FPM } & = & \text { fiber placement machine } \\ \text { VS } & = & \text { variable stiffness }\end{array}$

\section{Introduction}

In conventional composite laminate design the desire is to find the arrangement of material constituents that best satisfies the posted requirements on, for instance, strength, stiffness and cost. Fiber orientation angles are usually chosen from the set $\left\{0^{\circ} /+45^{\circ} /-45^{\circ} / 90^{\circ}\right\}$ and remain constant throughout an entire ply. Furthermore, laminate designs are limited to a fixed number of plies, $n$, and design rules like the 10\%-rule. This limits the scope of the optimization problem, and the directional properties of the composite material cannot be used to their full extend, for there are only a limited number of possible combinations. Hence there is room for improvement in terms of mechanical properties and/or weight of the structure. Such improvement can be achieved by modification of the stacking sequence within the bounds of conventional design rules, i.e. laminate tailoring. As defined by Jones ${ }^{1}$ laminate tailoring is the act of meeting "...particular structural requirements with little waste of material capability."

The concept of laminate tailoring can be taken further by expanding the range of fiber orientation angles used to design a structure and by allowing fiber angle variation within a ply. The latter form of composite laminate tailoring is known as variable stiffness (VS) composite laminate design, and will form the focus of this paper. It is posited by Jegley et al. ${ }^{2}$ that matching the spatial variation of the stress state in a structure by varying the fiber orientation angle, exploits the direction material properties of fiber-reinforced composite laminates more efficiently, for both strength and stiffness. The variation in fiber orientation angle will result in stiffness properties being spatially variable, hence the term VS composite laminate.

A considerable amount of work on VS composite structures can be found in literature. VS design has been applied to a range of design studies: buckling optimization, ${ }^{2-8}$ maximization of eigenfrequencies,${ }^{9,10}$ in-plane compliance ${ }^{11,12}$ and strength. ${ }^{13,14}$ Without exception significant improvements in performance over constant stiffness (CS) designs are reported.

Allowing stiffness variation in composite design leads to a significant increase of the number of design variables. Using fiber angles as design variables inevitably leads to an ill-behaved objective function with many local minima. ${ }^{15}$ The number of variables describing the mechanical properties of a laminate can be successfully reduced to twelve dimensionless variables by using lamination parameters (LP), as introduced by Tsai and Hahn. ${ }^{16}$ Imposing balance and symmetry, the mechanical properties of a laminate can be described by as little as four dimensionless variables. Most lay-up optimization problems can be made convex by choosing LPs as design variables. ${ }^{17}$ This makes it attractive to design VS structures in terms of LPs, see for instance the work by Setoodeh et al. ${ }^{6}$ and IJsselmuiden et al..$^{8,12,18}$

A method to convert a VS laminate design in terms of LPs into a VS laminate design in terms of fiber angles is proposed by Van Campen et al. ${ }^{19}$ This method includes a constraint on in-plane fiber radius for production reasons. Automated fiber placement (AFP) is an enabling technology used for in-plane fiber steering, and was the manufacturing technology of choice used in the work presented here. There is a minimum to the in-plane steering radii that can be accomplished using AFP. Fiber steering also may result in thickness-build up due to overlapping fiber paths, ${ }^{20}$ which is discussed in this paper.

The aforementioned method to convert VS designs in terms of LPs into fiber angle designs returns fiber orientation angles at the nodes of the finite element model describing the structure being studied. Fiber courses of specified width and starting location are required for a fiber placement machine (FPM) to be able to manufacture fiber steered laminates. Hence the nodal fiber angle distribution needs to be converted into 
fiber paths, on the basis of which a discrete number of course locations can be determined. A method to do so based on streamline analogy is posited by Blom et al. ${ }^{21}$

The main subject of this paper is the conversion of VS designs defined in terms of LPs into fiber-steered VS composite laminate designs. Hereto, first the design of VS composite structures using LPs and the subsequent conversion into a design in terms of fiber angles will be discussed. The reader will be familiarized with the method to covert LPs into fiber angles posited by Van Campen and Gürdal. ${ }^{19}$ The effect of this conversion method on the thickness build-up due to fiber steering is discussed in this paper. The streamline analogy presented by Blom et al. ${ }^{21}$ will be used to obtain an estimate of the smeared thickness and to determine fiber paths for each layer in the laminate, and it will be shown how this estimate can be used as a constraint in the conversion process. The effect of in-plane fiber curvature on thickness build-up will be demonstrated using a square plate under a biaxial compressive load for which the optimum LP distribution is assumed to be known. The fiber angle distributions and fiber paths for a number of constraints on in-plane curvature will be presented.

\section{Two-Step Optimization of Variable Stiffness Laminates}

A consequence of designing a laminate in terms of LPs is that the obtained optimum design must be converted into a laminate configuration. The complete design process proposed for VS laminate design and optimization is shown in figure 1. In the following both the continous design in terms of LPs and the discrete optimization in terms of fiber angles will be discussed.

\section{A. Optimization of VS composite laminates using LPs}

Lamination parameters (LP), introduced by Tsai and Pagano ${ }^{22}$ and Tsai and Hahn, ${ }^{16}$ provide a convenient means to describe the properties of any laminated stack in a non-dimensional way based on classical lamination theory (CLT). The number of design variables involved in the optimization can be reduced to twelve for the case no assumptions are made about the laminate's build-up, and can be as low as four in case balanced symmetric laminates are considered. A brief overview of lamination parameters is presented in appendix A.

Using the work of Svanberg ${ }^{17}$ it can be explained how changing to LPs as design variables helps conditioning the layup optimization problem. IJsselmuiden
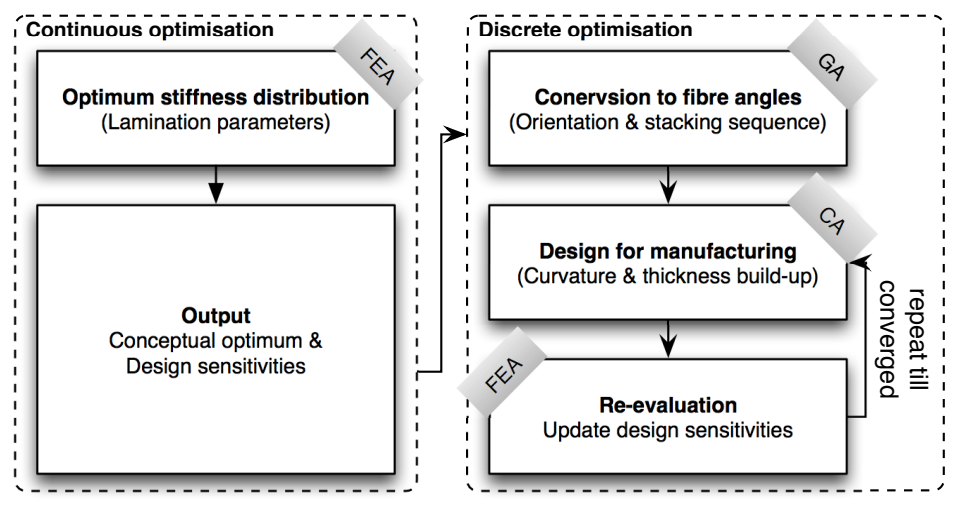
et $a l^{8}{ }^{8}$ exploit this trait to formulate a

Figure 1. Proposed process flow for optimization of VS laminates. reciprocal approximation scheme to determine the buckling load of composite panels; by expanding the buckling load factor linearly in terms of the in-plane stiffness and in terms of inverse bending stiffness, they obtain an approximation scheme that is convex in LP space. With this new scheme for a square plate loaded in biaxial compression a buckling load $52 \%$ better than the best known constant stiffness design was found. The optimized lamination parameters are plotted in figure 2 .

\section{B. Conversion from LPs to Fiber Angles}

The relationship between LPs and stacking sequence is not always unique ${ }^{23}$ there is no closed form solution to convert a point in LP space into a balanced symmetric stacking sequence. Even more so, when prescribing the number of layers in the laminate, certain points in LP space do not have a corresponding lay-up, and the closest alternative must be found. In previous work ${ }^{19}$ the conversion process was formulated as an optimization problem. Laminate design in terms of fiber angles in most cases is a non-convex optimization 


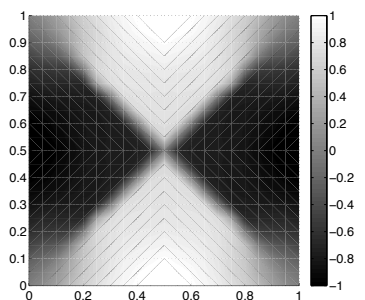

(a) $V_{1}^{\mathbf{A}}$

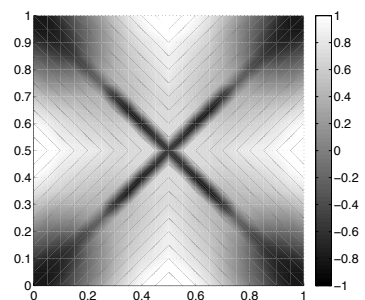

(b) $V_{3}^{\mathbf{A}}$

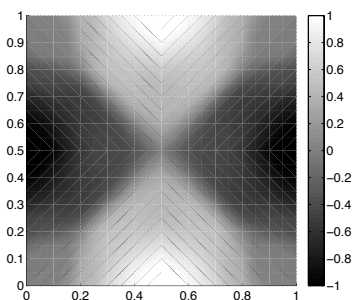

(c) $V_{1}^{\mathbf{D}}$

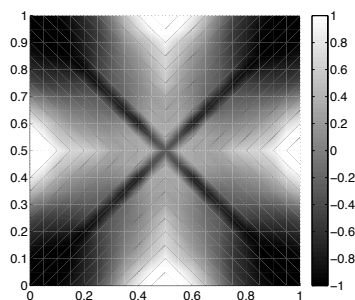

(d) $V_{3}^{\mathbf{D}}$

Figure 2. Optimal lamination parameters distribution for the square plate loaded in biaxial compression. The plotted squares represent the square panel, the value of the lamination parameters is indicated by the shading and ranges from -1 to 1 .

problem. ${ }^{17}$ Therefore a hybrid method was applied in which a GA was used to seed a gradient-based optimizer. The objective function that was used for this optimization was the least square distance of the LPs to the desired optimum point:

$$
f=\left|\mathbf{V}^{*}-\mathbf{V}\right| \quad \text { with } \quad \mathbf{V}=\left\{V_{1}^{\mathbf{A}}, V_{3}^{\mathbf{A}}, V_{1}^{\mathbf{D}}, V_{3}^{\mathbf{D}}\right\}
$$

where * denotes the given optimum lamination parameters.

Applied to a nodal distribution of LPs this conversion process results in a least-squares optimal nodal distribution of stacking sequences, which was used as a starting point for further optimization using a cellular automaton (CA) to include a constraint on in-plane curvature. The flow diagram of the CA is given in Fig. 3. Jacobi iteration was used to conserve the symmetry of the results. In order to facilitate the implementation of constraints in the CA an augmented objective function is proposed:

$$
\Phi=\left\{\begin{array}{lll}
f+\varepsilon g_{\max } & \text { if } & g_{\max } \leq 0 \\
f+\beta g_{\max } & \text { if } & g_{\max }>0
\end{array}\right.
$$

where $\Phi$ is to be minimized. Eq. 2 is an augmented objective function, in which $f$ describes the actual objective of the optimization problem, see Eq. $1, g$ are the constraint margins, $g=0.1$ means a $10 \%$ constraint violation, whereas $g=-0.1$ indicates a $10 \%$ constraint margin. They are multiplied by bonus term $\varepsilon$, respectively penalty term $\beta$. The constraint margin for in-plane fiber angle curvature, defined as $\kappa=|\nabla \theta|$, can be defined as:

$$
g_{\kappa}=\frac{\kappa}{\kappa_{\text {all }}}-1
$$

An update rule following Newton's method was proposed. At node $k$ this update rule takes the form:

$$
\theta^{(k+1)}=\theta^{(k)}-\alpha_{k} M_{k} \mathbf{g}_{k}
$$

where $\alpha_{k}$ is the allowed step size and:

$$
M_{k}=\left(H_{k}+\mu_{k} I\right)^{-1}
$$

with $\mu_{k}$ chosen such that the matrix $M_{k}$ is always positive definite. In the case where the Hessian is not positive definite, Newton's method will move to higher function values and might stall near singular points. In such a case stabilization is required. Alternatively, when no Hessian is available, or expensive to compute, one can switch to gradient descent, i.e. $H=I$ and $\mu_{k}=0$.

Given the linearity of the augmented objective function, Eq. 2 its Jacobian and Hessian can be decomposed in the Jacobians and Hessians of its respective parts. This is a useful feature as more constraints are imposed on the system.

The proposed update rule may not converge when an infeasible starting point is used. Therefore, the fiber angle design that was used to seed the CA was first regularized until $g \max$ was smaller than a predefined value. Regularization took place using a simple averaging rule. 


\section{Thickness Build-up}

Multiple tow courses are needed to construct a fiber-placed laminate. Here one layer of such a laminate is considered. Assuming the tow courses in this layer are all derived from one reference curve, there are multiple options to fill the entire layer with tow courses two of which will be considered here: (i) parallel paths, or (ii) shifted paths. ${ }^{24}$

The advantage of parallel paths is, that no gaps or overlaps between tows will occur, which results in a structure of uniform thickness and theoretically without any defects. A disadvantage is that the steering radii of each parallel tow are different, which at sufficient distance from the reference path will result in violation of the maximum allowable in-plane curvature, as is demonstrated in Fig. 4(a).

The in-plane curvatures of each tow course are equal when they are placed along a path which has been shifted parallel to the reference tow-path, as is demonstrated in Fig. 4(b). The parallel shift results in the combination of the two most extreme cases: (i) tows fully overlapping, or (ii) gaps between the tows with no overlaps. These gaps and overlaps cause a change in thickness of the layer under consideration, and thus of the laminate as a whole.

As a first approximation of thickness buildup due to fiber steering, gaps and overlaps can be smeared out over the surface of the struc-

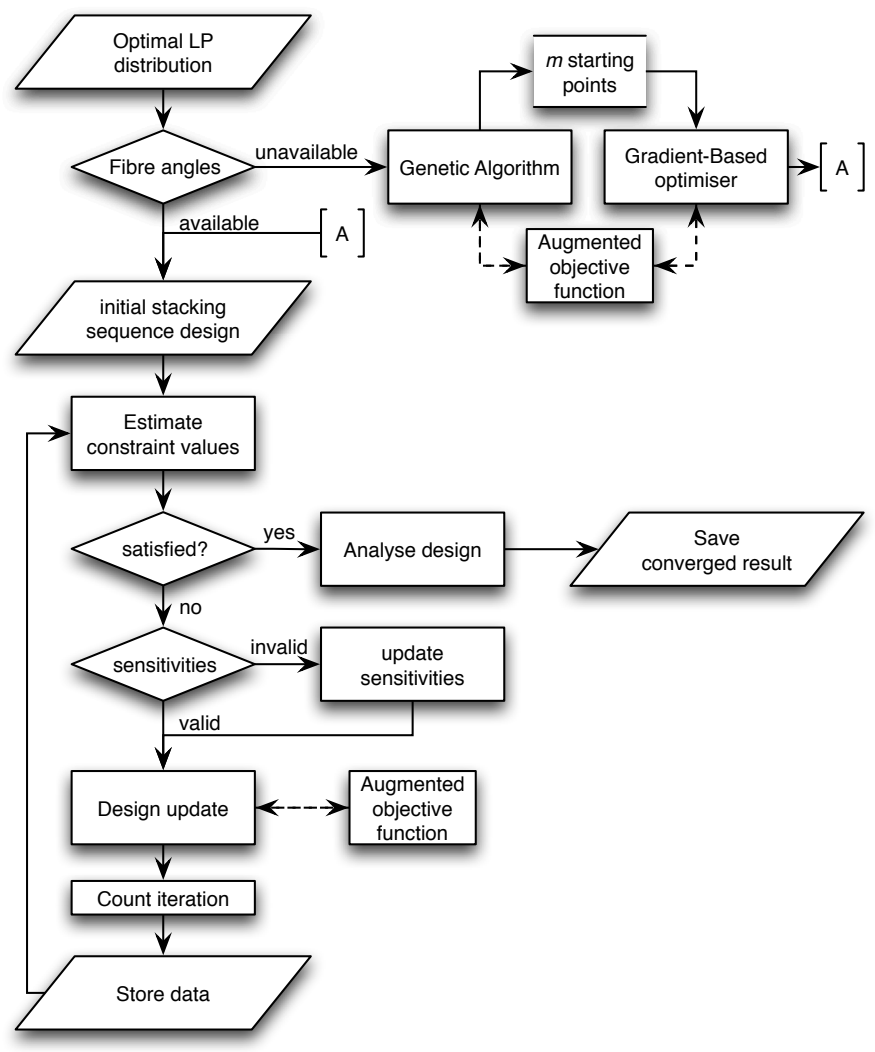

Figure 3. Flow diagram of the proposed Jacobi iteration scheme

ture, since their exact locations are still unknown. The streamline analogy can be used to estimate the smeared thickness of in-plane fiber angle distribution ${ }^{21}$ per ply:

$$
-\mathbf{s} \nabla(\ln t)=\mathbf{n} \nabla \theta
$$

where $\mathbf{s}$ is the tangent vector in the direction of the fiber and $\mathbf{n}$ is the vector in the plane of the lamina normal to the direction of the fiber. By change of variable Eq. 6 can be written as:

$$
-\mathbf{s} \nabla \tau=\mathbf{n} \nabla \theta
$$

where $\tau=\ln t$. For a fiber angle variation given on a nodal grid this results in a system of equations. Making use of the linearity of all equations this system can be rearranged in the form:

$$
\mathbf{M} \tau=\mathbf{b}
$$

Boundary conditions can enter this equation through vector $\mathbf{b}$. Uniform thickness at the inflow boundaries was assumed for the thickness distributions presented in this paper. The hyperbolic differential equation, Eq. 8, can be solved by a finite element scheme. ${ }^{25}$ For the work presented in this paper the QUADprog function in MATLAB ${ }^{\circledR}$ was used. The fiber course designs were optimized for smoothness, i.e. the differences in thickness between nodes were minimized.

Once the thickness distribution is known, the streamline analogy can be used to determine fiber paths. Mathematically a stream function is defined as: ${ }^{21}$

$$
\Psi(x, y)=C
$$

which connects all points for which $C$ is equal. The solution for the stream function depends on the provided boundary conditions. Note, that the streamlines for $+\theta$ and $-\theta$ will be different. 


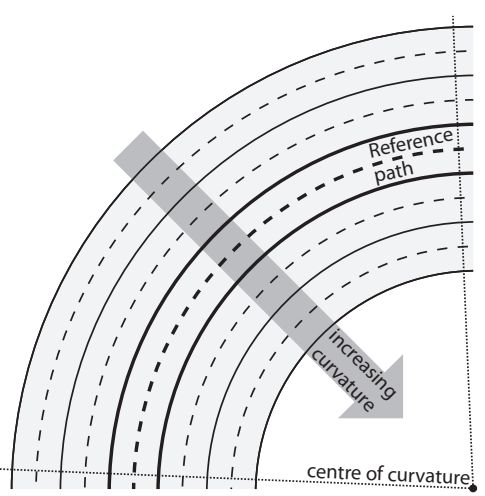

(a) Parallel paths

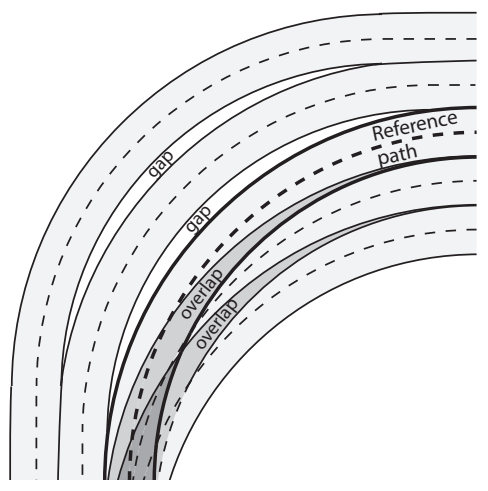

(b) Shifted paths

Figure 4. Tow paths derived from a reference curve.

\section{Results and Discussion}

The square plate of 1 by 1 meter in dimension is shown in figure 5 . The plate is loaded in $x$-direction by a uniformly distributed compressive load. The buckling load of the plate was maximized in a previous design step using lamination parameters (LP) as design variables. ${ }^{8}$ For this optimization a $1.524 \mathrm{~mm}$-thick (0.06in.-thick) laminate of the following material properties was considered: $E_{1}=181 \mathrm{GPa}(26.25 \mathrm{Msi}), E_{2}=10.3 \mathrm{GPa}$ (1.49Msi), $G_{12}=7.17 \mathrm{GPa}(1.04 \mathrm{Msi})$ and $\nu_{12}=0.28$. The optimized LP-distribution is presented in figure 2 .

The effect of the in-plane curvature constraint on the maximum thickness build-up in the panel was studied. To this end, the allowable maximum curvature was varied from $1 \mathrm{~m}^{-1}$ to $100 \mathrm{~m}^{-1}$ for the layup $\left[ \pm \theta_{1} / \pm \theta_{2} / \pm \theta_{3} / \pm \theta_{4}\right]_{s}$. The step-size of the $\mathrm{CA}$ was set to $1 \cdot 10^{-5}$ and the optimization was considered to have converged after 50 iterations with less than $0.1 \%$ improvement. Furthermore the fiber angle seed was regularized until $g_{\max } \leq-0.05$.

The results obtained for maximum thickness, objective function value

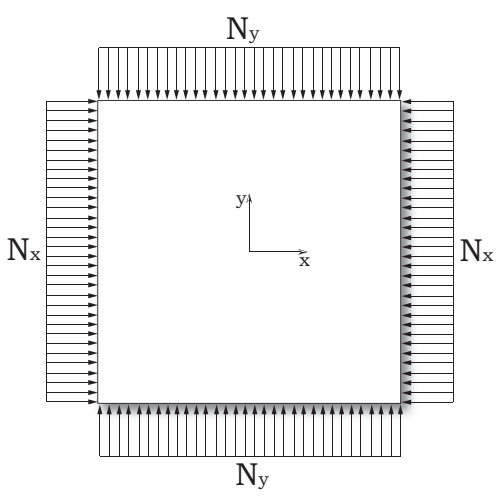

Figure 5. Square panel loaded by biaxial distributed compressive load. and buckling load are plotted in Fig. 6 . The curvature value on the $x$-axis
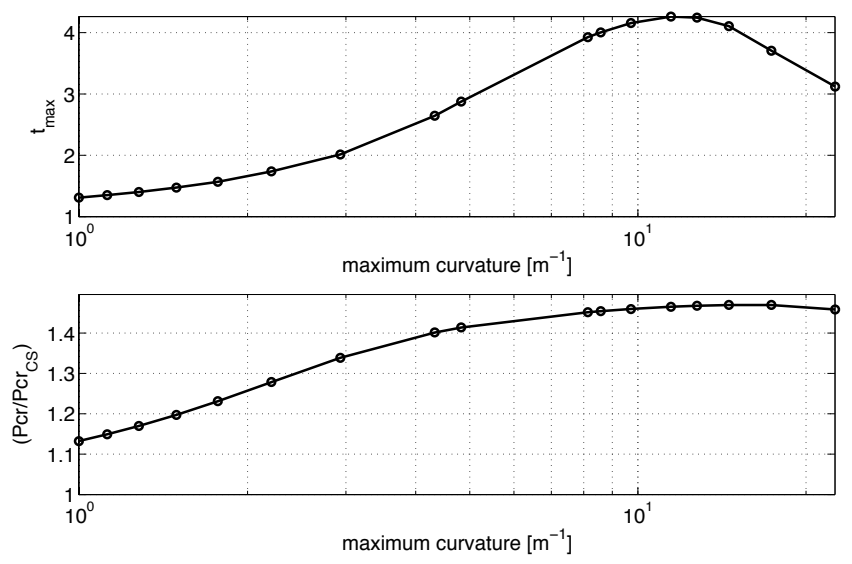

Figure 6. Maximum smeared thickness normalized by the thickness of the original panel and buckling load as function of the constraint on in-plane curvature. 
is the actual value attained by the structure, not the constrained value. The unconstrained design has a buckling load that is $46 \%$ better than the best CS design. In comparison the optimized LP distribution, shown in Fig. 2, has a buckling load $52 \%$ higher than the best CS design. These values were obtained assuming constant thickness of the panel, i.e. thickness build-up due to fiber steering was ignored in the buckling analysis.

The relationship between the curvature constraint and thickness build-up appears to be similar to that between the curvature constraint and the buckling load of the panel, with one main exception: the thickness build-up for the unconstrained design is smaller than for the design where fiber angle curvature is just constrained. In general, both the buckling load and maximum thickness of the panel are reduced when $\kappa$ is restrained to smaller values. It is concluded that a constraint on maximum in-plane curvature helps to constrain thickness build-up in the panel.

Fiber angle designs were obtained for the unconstrained case, and for the case where $\kappa_{\text {all }}=4.878 \mathrm{~m}^{-1}$. The results are plotted in Figs. 7 and 8. The buckling loads for these designs were respectively $46 \%$ and $31 \%$ better than the best known constant stiffness design.

The fiber paths for the unconstrained result, clearly showed the necessity fro a constraint on in-plane curvature, the fiber paths appeared impossible to manufacture and thickness build-up was deemed unrealistic. Large parts of the panel were not covered by any fiber paths, or only very few. The shading used in Fig. 7 to depict the thickness build indicates a smeared thickness of 0 at most locations in panel. This explains the strongly reduced maximum thickness found for the unconstrained case for the study on the effect of in-plane curvature on thickness build-up.

The obtained fiber paths for the constrained case, $\kappa_{\text {all }}=4.878 \mathrm{~m}^{-1}$, appeared to be smooth. Thickness build-up was found to be most dominant at the corners of the panel, the streamlines were closer together in these areas. Note that the fiber paths for $+\theta$ and $-\theta$ are different.

\section{Concluding Remarks}

A design methodology for variable stiffness (VS) composite laminates was discussed in this paper. Part of this methodology is the conversion from lamination parameters (LP) to fiber angles. A previous study by the authors on the conversion of LPs into fiber angles was used for this step. A constraint on in-plane fiber curvature, a manufacturing constraint for automated fiber placement (AFP), was already included in this conversion method. In the work presented here the effect of this curvature constraint on the thickness build-up that will occur due to fiber steering is considered. An estimate of the smeared thickness buildup was obtained for each layer in the laminate by solving a hyperbolic differential equation. Using the streamline analogy, fiber paths were determined for the obtained thickness distributions. Uniform inflow was considered, and designs were maximized for smoothness. A square plate loaded in biaxial compression was used to demonstrate the effect of the in-plane curvature constraint on thickness build-up, and several fiber angle designs, thickness distributions and fiber paths were given for this structure.

The results showed, that thickness build-up due to fiber steering strongly depends on the maximum in-plane curvature that is allowed. Study of an unconstrained fiber angle design and a fiber angle design constrained in terms of curvature revealed the need for a curvature constraint if manufacturable designs are to be obtained. In future research a constraint on thickness build-up due to fiber steering will be included in the conversion process, and the effect of thickness build-up will be included in the buckling analysis.

\section{Acknowledgements}

The AUTOW project's, part of the European Union Sixth Framework Programme, support of this work is gratefully acknowledged. The first author would like to thank Samuel IJsselmuiden for providing him with the input lamination parameter distribution used for the square plate example and for the code he provided to allow the first author to perform the required buckling analysis. 


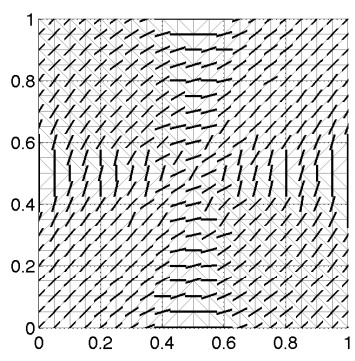

(a) $\theta_{1}$

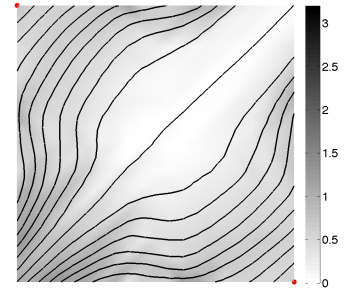

(e) $+\theta_{1}$

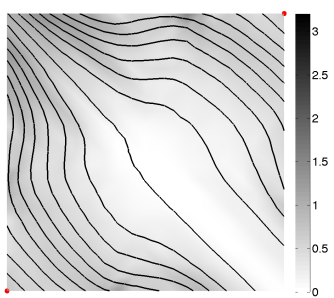

(i) $-\theta_{1}$

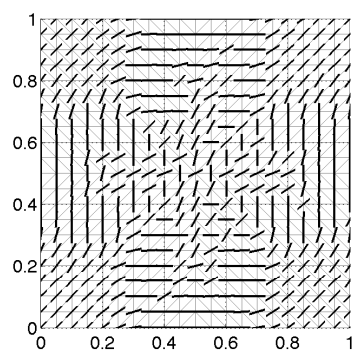

(b) $\theta_{2}$

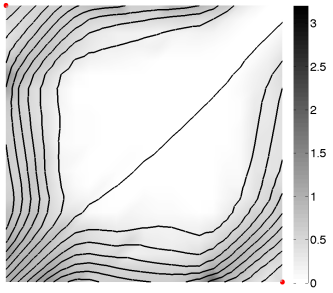

(f) $+\theta_{2}$

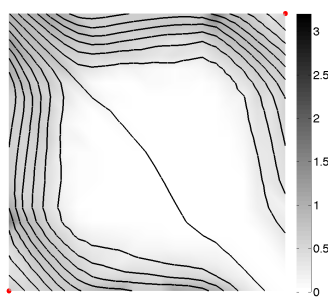

(j) $-\theta_{2}$

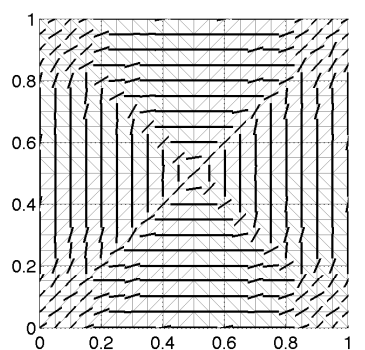

(c) $\theta_{3}$

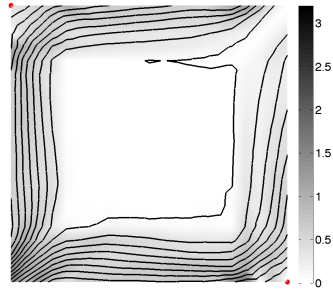

(g) $+\theta_{3}$

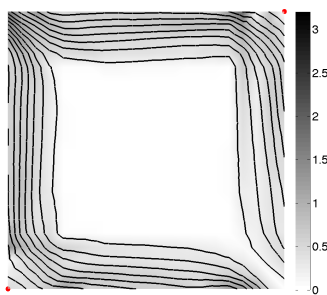

(k) $-\theta_{3}$

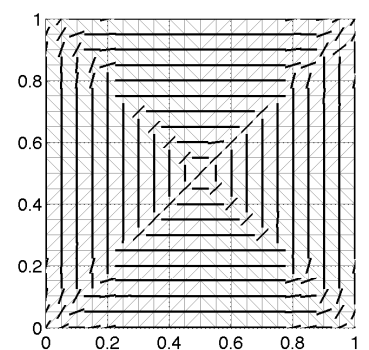

(d) $\theta_{4}$

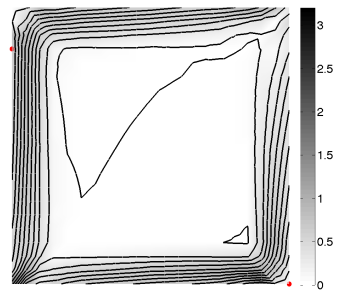

(h) $+\theta_{4}$

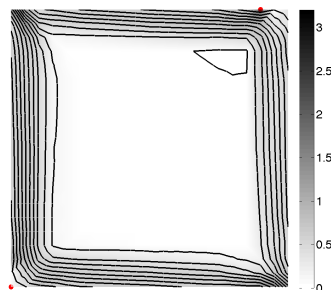

(l) $-\theta_{4}$

Figure 7. Fiber angles streamlines without constraint on in-plane curvature. Thickness build-up is indicated by the shading. 


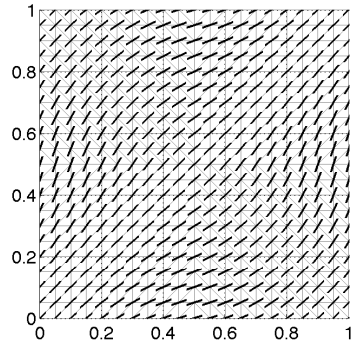

(a) $\theta_{1}$

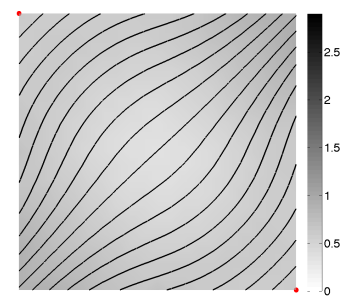

(e) $+\theta_{1}$

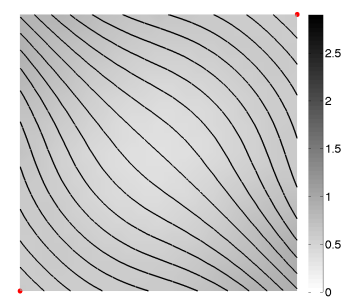

(i) $-\theta_{1}$

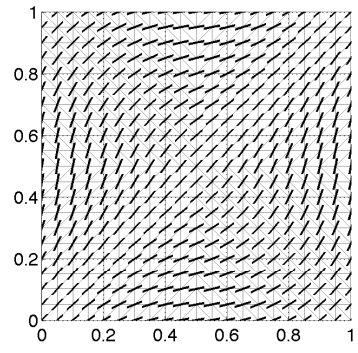

(b) $\theta_{2}$

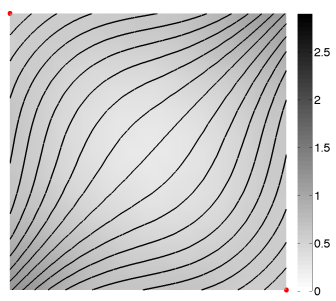

(f) $+\theta_{2}$

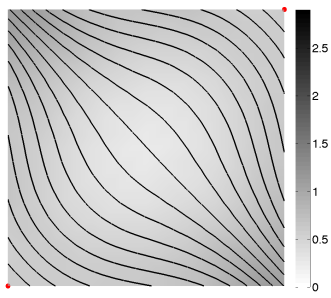

(j) $-\theta_{2}$

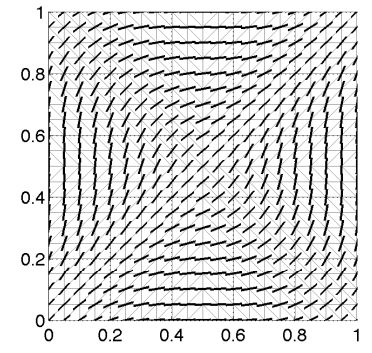

(c) $\theta_{3}$

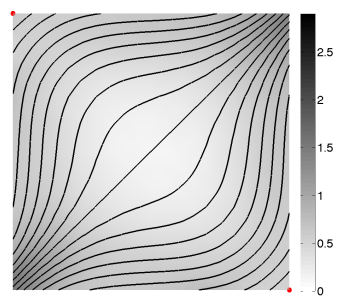

(g) $+\theta_{3}$

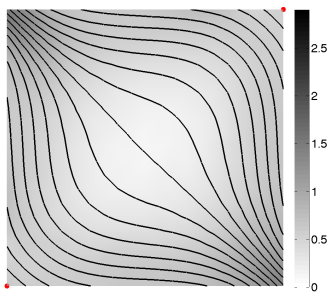

(k) $-\theta_{3}$

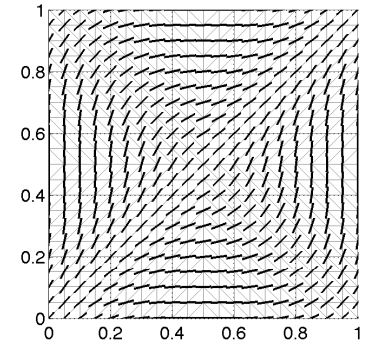

(d) $\theta_{4}$

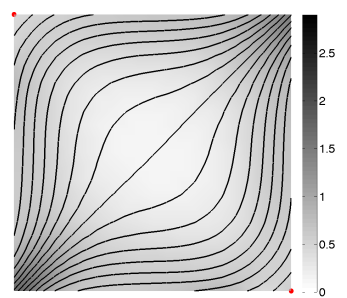

(h) $+\theta_{4}$

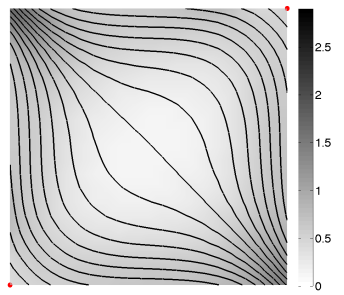

(1) $-\theta_{4}$

Figure 8. Fiber angles and streamlines for $\kappa_{\text {all }}=4.878 \mathrm{~m}^{-1}$. Thickness build-up is indicated by the shading. 


\section{References}

${ }^{1}$ Jones, R. M., Mechanics of Composite Materials, Taylor and Francis, Philadelphia, second edition ed., 1999.

${ }^{2}$ Jegley, D. C., Tatting, B. F., and Gürdal, Z., "Optimization of elastically tailored tow-placed plates with holes," $44^{\text {th }}$ AIAA/ASME/ASCE/AHS Structures, Structural Dynamics, and Materials Conference, American Institute of Aeronautics and Astronautics, Norfolk, VA, 7 - 10 April 2003.

${ }^{3}$ Hyer, M. and Lee, H., "The Use of Curvilinear Fiber Format to Improve Buckling Resistance of Composite Plates with Central Circular Holes," Composite Structures, Vol. 18, No. 3, 1991, pp. 239-261.

${ }^{4}$ Gürdal, Z. and Olmedo, R., "In-Plane Response of Laminates with Spatially Varying Fiber Orientations: Variable Stiffness Concept," AIAA Journal, Vol. 31, No. 4, April 1993, pp. $751-758$.

${ }^{5}$ Wu, K. C., Gürdal, Z., and Starnes, Jr., J. H., "Structural Response of Compression-Loaded,Tow-Placed, Variable Stiffness Panels," No. AIAA 2002-1512, Proceedings of the $43^{\text {rd }}$ AIAA/ASME/ASCE/AHS/ASC Structures, Structural Dynamics and Materials Conference, Denver, CO, 22-25 April 2002.

${ }^{6}$ Setoodeh, S., Abdalla, M. M., IJsselmuiden, S. T., and Gürdal, Z., "Design of variable-stiffness composite panels for maximum buckling load," Composite Structures, Vol. 87, No. 1, January 2009, pp. 109-117.

${ }^{7}$ Abdalla, M. M., Gürdal, Z., and Abdelal, G. F., "Thermomechanical Response of Variable Stiffness Composte Panels," Journal of Thermal Stresses, Vol. 32, January 2009, pp. 187 - 208.

${ }^{8}$ IJsselmuiden, S. T., Abdalla, M. M., and Gürdal, Z., "Optimization of Variable-Stiffness Panels for Maximum Buckling Load Using Lamination Parameters," AIAA Journal, Vol. 48, No. 1, January 2010, pp. 134 - 143.

${ }^{9}$ Blom, A. W., Tatting, B. F., Hol, J. M. A. M., and Gürdal, Z., "Path Definintions for Elastically Tailored Conical Shells," No. AIAA 2006-1940, Proceedings of the $47^{\text {th }}$ AIAA/ASME/ASCE/AHS/ASC Structures, Structural Dynamics, and Materials Conference, Newport, RI, USA, 1 - 4 May 2006.

${ }^{10}$ Blom, A. W., Setoodeh, S., Hol, J. M. A. M., and Gürdal, Z., "Design of variable-stiffness concial shells for maximum fundamental eigenfrequency," Computers \& Structures, Vol. 86, 2008, pp. $870-878$.

${ }^{11}$ Honda, S. and Narita, Y., "Design of Composite Plate with Optimally Distributed Short Fibers," 16 th International Conference on Composite Materials, Kyoto, Japan, May 2007.

${ }^{12}$ IJsselmuiden, S. T., Abdalla, M. M., Pilaka, V. K., and Gürdal, Z., "Design of Variable Stiffness Composite Structures for Advanced Fibre Placement Technology," SAMPE 2010, New Materials and Processes for a New Economy, 17 - 20 May 2010.

${ }^{13}$ Alhajahmad, A., Abdalla, M. M., and Gürdal, Z., "Design Tailoring for Pressure Pillowing Using Tow-Placed Steered Fibers," Journal of Aircraft, Vol. 45, No. 2, March - April 2008, pp. 630 - 640.

${ }^{14}$ Blom, A. W., Lopes, C. S., Kromwijk, P. J., Gürdal, Z., and Camanho, P., "A Theoretical Model to Study the Influence of Tow-drop Areas on the Stiffness and Strength of Variable-stiffness Laminates," Journal of Composite Materials, Vol. 43, No. 5, 2009, pp. $403-425$.

${ }^{15}$ Conti, P., Luparello, S., and Pasta, A., "Layer thickness optimisation in a laminated composite," Composites Part B: Engineering, Vol. 28B, 1997.

${ }^{16}$ Tsai, S. W. and Hahn, H. T., Introduction to Composite Materials, Technomic Publishing Co., Inc., 1980.

${ }^{17}$ Svanberg, K., "On local and global minima in structural optimization," New Directions in Optimum Structural Design (Proceedings of the Second International Symposium on Optimum Structural Design), Tuscon, AZ, 1984.

${ }^{18}$ IJsselmuiden, S. T., Abdalla, M. M., and Gürdal, Z., "Implementation of Strength-Based Failure Criteria in the Lamination Parameter Design Space," AIAA Journal, Vol. 46, No. 7, July 2008, pp. 1826 - 1834.

${ }^{19}$ van Campen, J. M., Kassapoglou, C., and Gürdal, Z., "Generating Realistic Laminate Fiber Angle Distributions for Optimal Variable Stiffness Laminates," Submitted to Composites Part B: Engineering, Ref. No.: JCOMB-D-11-00115.

${ }^{20}$ Setoodeh, S., Blom, A. W., Abdalla, M. M., and Gürdal, Z., "Generating Curvilinear Fiber Paths from Lamination Parameters Distribution," AIAA-2006-1875, 47th AIAA/ASME/AHS/ASC Structures, Structural Dynamics and Material Conference, 2006.

${ }^{21}$ Blom, A. W., Abdalla, M. M., and Gürdal, Z., "Optimization of course locations in fiber-placed panels for general fibre angle distributions," Composites Science and Technology, Vol. 70, No. 4, April 2010, pp. 564 - 570.

${ }^{22}$ Tsai, S. W. and Pagano, N. J., Invariant properties of composite materials, composite material workshop, Technomic, 1968.

${ }^{23}$ Diaconu, C. G., Sato, M., and Sekine, H., "Feasible Region in General Design Space of Lamination Parameters for Laminated Composites," AIAA Journal, Vol. 40, No. 3, March 2002, pp. $559-565$.

${ }^{24}$ Tatting, B. F. and Gürdal, Z., "Automated Finite Element Analysis of Elastically-Tailored Plates," Contractor Report NASA/CR-2003-212679, National Aeronautics and Space Administration, 2003.

${ }^{25}$ Pilaka, V. K., Design of Realistic Variable Stiffness Composites, Master's thesis, Delft Universtiy of Technology, 2010. 


\section{A. Lamination Parameters}

The in-plane and out-of-plane lamination parameters(LP), introduced by Tsai and Pagano ${ }^{22}$ and Tsai and Hahn, ${ }^{16}$ are respectively defined as:

$$
\begin{array}{r}
\left(V_{1}^{\mathbf{A}}, V_{2}^{\mathbf{A}}, V_{3}^{\mathbf{A}}, V_{4}^{\mathbf{A}}\right)=\int_{-\frac{1}{2}}^{\frac{1}{2}}(\cos 2 \theta(\bar{z}), \sin 2 \theta(\bar{z}), \cos 4 \theta(\bar{z}), \sin 4 \theta(\bar{z})) d \bar{z} \\
\left(V_{1}^{\mathbf{D}}, V_{2}^{\mathbf{D}}, V_{3}^{\mathbf{D}}, V_{4}^{\mathbf{D}}\right)=12 \int_{-\frac{1}{2}}^{\frac{1}{2}} \bar{z}^{2}(\cos 2 \theta(\bar{z}), \sin 2 \theta(\bar{z}), \cos 4 \theta(\bar{z}), \sin 4 \theta(\bar{z})) d \bar{z}
\end{array}
$$

where $\bar{z}=\frac{z}{h}$ is the normalized through-the-thickness coordinate of the layers, $h$ is the total thickness of the laminate, and $\theta(\bar{z})$ is the fiber angle at $\bar{z}$. The in-plane laminate stiffness matrix $\mathbf{A}$ and the out-of-plane laminate stiffness matrix $\mathbf{D}$ then become linear functions of the lamination parameters as follows:

$$
\begin{array}{r}
\mathbf{A}=h\left(\Gamma_{0}+V_{1}^{\mathbf{A}} \Gamma_{1}+V_{2}^{\mathbf{A}} \Gamma_{2}+V_{3}^{\mathbf{A}} \Gamma_{3}+V_{4}^{\mathbf{A}} \Gamma_{4}\right) \\
\mathbf{D}=\frac{3 h^{3}}{12}\left(\Gamma_{0}+V_{1}^{\mathbf{D}} \Gamma_{1}+V_{2}^{\mathbf{D}} \Gamma_{2}+V_{3}^{\mathbf{D}} \Gamma_{3}+V_{4}^{\mathbf{D}} \Gamma_{4}\right)
\end{array}
$$

where the $\Gamma_{i}\left(i=(1, \ldots, 4)\right.$ matrices in terms of the material invariants? ${ }^{2}$ are given by:

$$
\begin{gathered}
\Gamma_{0}=\left[\begin{array}{ccc}
U_{1} & U_{4} & 0 \\
U_{4} & U_{1} & 0 \\
0 & 0 & U_{5}
\end{array}\right], \Gamma_{1}=\left[\begin{array}{ccc}
U_{2} & 0 & 0 \\
0 & -U_{2} & 0 \\
0 & 0 & 0
\end{array}\right], \Gamma_{2}=\left[\begin{array}{ccc}
0 & 0 & \frac{1}{2} U_{2} \\
0 & 0 & \frac{1}{2} U_{2} \\
\frac{1}{2} U_{2} & \frac{1}{2} U_{2} & 0
\end{array}\right] \\
\Gamma_{3}=\left[\begin{array}{ccc}
U_{3} & -U_{3} & 0 \\
-U_{3} & U_{3} & 0 \\
0 & 0 & -U_{3}
\end{array}\right], \Gamma_{4}=\left[\begin{array}{ccc}
0 & 0 & U_{3} \\
0 & 0 & -U_{3} \\
U_{3} & -U_{3} & 0
\end{array}\right]
\end{gathered}
$$

The material invariants, $U_{i}(i=1, \ldots, 5)$, are defined in terms of lamina reduced stiffnesses by:

$$
\begin{array}{r}
U_{1}=\frac{1}{8}\left(3 S_{11}+3 S_{22}+2 S_{12}+4 S_{66}\right) \\
U_{2}=\frac{1}{2}\left(S_{11}-S_{22}\right) \\
U_{3}=\frac{1}{8}\left(S_{11}+S_{22}-2 S_{12}-4 S_{66}\right) \\
U_{4}=\frac{1}{8}\left(S_{11}+S_{22}+6 S_{12}-4 S_{66}\right) \\
U_{5}=\frac{1}{8}\left(S_{11}+S_{22}-2 S_{12}+4 S_{66}\right)
\end{array}
$$

